\title{
Histopathology and in situ Hybridization of Coatomer (CopI) Knockdown in Western Corn Rootworm (Diabrotica virgifera virgifera)
}

\author{
Andrew J. Bowling ${ }^{1 *}$, Heather E. Pence ${ }^{1}$ and Elane Fishilevich ${ }^{2}$ \\ 1. Corteva Agriscience ${ }^{\mathrm{TM}}$ Agriculture Division of DowDuPont, Dow AgroSciences LLC, IN, USA. \\ 2. University of Nebraska-Lincoln, Department of Entomology, Lincoln, NE, USA. \\ * Corresponding author: andrew.bowling@corteva.com
}

The US is the world's largest producer of maize, with nearly 100 million acres allocated to maize production annually. Western corn rootworm (WCR; Diabrotica virgifera virgifera) is currently the major agronomically important pest of maize in the US Corn Belt. One of the most effective methods for protecting commercial maize crops from rootworm damage is through the incorporation of insect resistance proteins. Currently, a new class of insect resistance traits based on RNAi is being developed. RNAscope in situ hybridization (ISH) is a single molecule sensitivity detection method for localizing mRNA in tissue sections [1], and it was recently shown to be compatible with removable resin sections [1]. Resin preserves tissue ultrastructure with greater fidelity than traditional wax, and also allows for the use of thinner sections, both of which facilitate higher quality ISH analyses.

For histopathological studies, larvae were exposed to dsRNAs targeted to CopI $\alpha$, CopI $\beta$, or YFP (negative control). After 96 hours of feeding, larvae were fixed, infiltrated with LR White, and heat polymerized. Larvae were sectioned longitudinally at $500 \mathrm{~nm}$ thickness on a DiATOME histoknife, stained, and imaged. For in situ hybridization, larvae were collected after 48 hours on diet containing dsRNAs, fixed, infiltrated with 3:1 butyl:methyl methacrylate, and UV polymerized, all at $4^{\circ} \mathrm{C}$. Larvae were sectioned at $1 \mu \mathrm{m}$ thickness on a DiATOME histoknife, sections were deplasticized, and analyzed with the Advanced Cell Diagnostics (ACD) RNAscope VS Assay on a Ventana Discovery Ultra. Sections were imaged on a Leica DM5000B microscope and a Leica DFC 7000T camera.

The native expression patterns of CopI $\alpha$ and $\operatorname{CopI} \beta$ were found to be very similar, with the majority of the CopI mRNA in the midgut enterocytes and the cells of the Malpighian tubules (Figure 1A and 1C). Nearly complete knockdown of the mRNAs from both CopI genes was observed after 48 hours of feeding with gene-specific dsRNAs (Figure 1B and 1D). Furthermore, this knockdown was observed to be systemic, as evidenced by loss of CopI transcripts from non-alimentary system organs throughout the body of the larva. There was also loss of CopI transcripts from fat body adipocytes and cells of the ventral nerve cord, which are both organs distal to the alimentary system.

The most obvious histological impact of the loss of CopI transcripts on the morphology of WCR larvae was severe damage to the midgut enterocytes and constriction of the anterior midgut circular muscles (Figure 2C, 2D, 2E, and 2F). $\operatorname{CopI} \beta$ dsRNA seems to have caused slightly more damage to the AMG, and especially the MMG and PMG regions, than the CopI $\alpha$ dsRNA. More subtle changes were noted to the Malpighian tubules and hindgut, both of which were observed to be swollen following CopI dsRNA treatments, and the hemolymph volume appeared dramatically reduced (Figure 2C and 2E). No damage to the midgut was observed with YFP dsRNA feeding (Figure 2A and 2B). For both targets, the nearcomplete loss of the midgut enterocytes is likely lethal to WCR larvae, as the loss of midgut enterocytes and the constriction of circular muscles caused by knockdown of CopI is analogous to the effects seen with several types of insecticidal proteins [2]. 
References:

[1] Wang et al., Journal of Molecular Diagnostics 14 (2012), p. 22.

[2] Bowling et al., Toxins 9 (2017), p. 1.

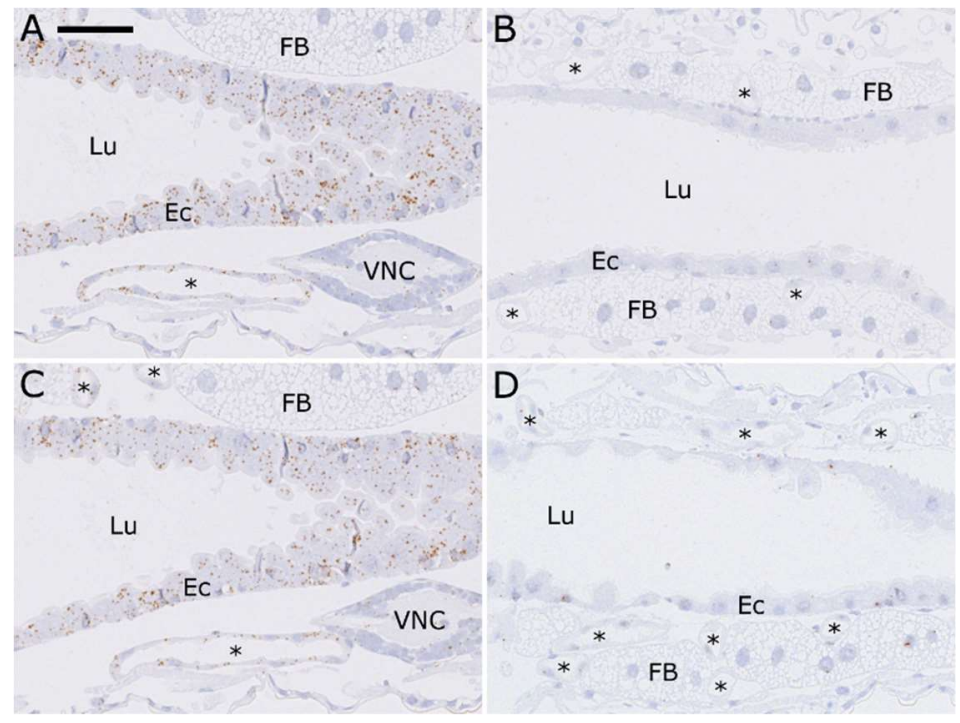

Figure 1. RNAscope ISH on longisections of WCR. Transcripts detected with DAB (brown), sections counterstained with hematoxylin. A.) Median section of a YFP dsRNA-fed (negative control) WCR larva showing CopI $\alpha$ transcripts most abundant in midgut enterocytes, and to a lesser extent in the Malpighian tubules. B.) Larva fed CopI $\alpha$ dsRNA, showing a dramatic reduction of CopIa mRNA in midgut and Malpighian tubule cells. C.) A median section of a negative control larva showing the native CopI $\beta$ expression pattern, which is very similar to CopI $\alpha$. D.) Larva fed CopIb dsRNA, showing a similar reduction of $\operatorname{CopI} \beta$ mRNA that was seen with CopI $\alpha . \mathrm{Ec}=$ midgut enterocytes; $\mathrm{VNC}=$ ventral nerve cord ganglion; $\mathrm{FB}=$ fat body; $\mathrm{Lu}=$ midgut lumen; * = Malpighian tubule. Scale bar $=50 \mu \mathrm{m}$.

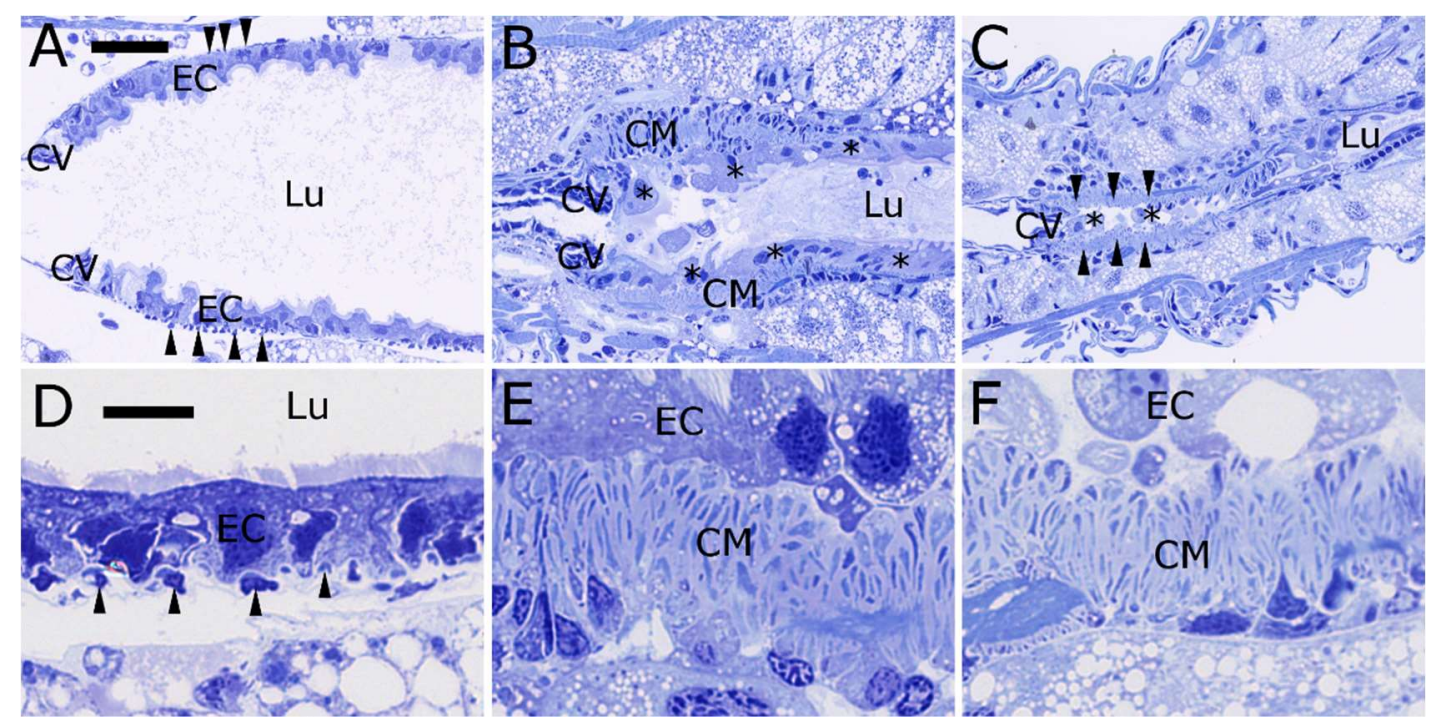

Figure 2. Histopathological impact of the loss of CopI $\alpha$ and $\operatorname{CopI} \beta$ mRNA. A. and D.) A larva fed YFP dsRNA, showing the normal appearance of the midgut. Larvae fed CopI $\alpha$ dsRNA (B and E) or CopI $\beta$ dsRNA ( $\mathrm{C}$ and $\mathrm{F}$ ), showing dramatic damage to the anterior midgut cells, including lysis and shedding of the enterocytes and midgut stem cells, severe contraction of the circular muscle bands, and near-total closure of the midgut lumen. $\mathrm{Lu}=$ lumen; Ec = enterocytes; $\mathrm{CM}$ and arrowheads = circular muscles, * = enterocyte remnants. Scale bar A, B, C $=50 \mu \mathrm{m} ; \mathrm{D}, \mathrm{E}, \mathrm{F}=10 \mu \mathrm{m}$ 